\title{
A Mathematical Model of Political Rumour in Assakio Community with Conditional Latent Period in a Varying Population
}

\author{
Atanyi Yusuf Emmanuel ${ }^{1}$ Imam Akayede ${ }^{2}$., A. S Olagunju ${ }^{3}$. \\ Gilbert Aimufua ${ }^{4}$ Apene Oghenevovwero Zion ${ }^{5}$. \\ Umar Sani ${ }^{6}$., S. H. Isah ${ }^{7}$ \\ 1, 2 \&3 Department of Mathematics, \\ Federal University of Lafia, \\ PMB 146, \\ Lafia, Nigeria \\ ${ }^{4 \& 5}$ Department of Computer \\ Nasarawa State University, \\ Keffi, \\ PMB 1022 Keffi \\ Nigeria \\ ${ }^{687}$ Department of mathematics \\ Nasarawa State University, Keffi, \\ PMB 102 \\ Keffi Nigeria
}

\begin{abstract}
In this paper a rumour propagation model with conditional latent period and varying population is considered. In the literature, classical model assume that an ignorant individual enters the latent period and decide whether to become a spreader or stifler. In our model we introduce a new compartment called the blackmailers, another type of spreaders who spread the rumour for selfish reason. The model equations are first transformed into proportions, thus reducing the model equations from five to four differential equations. The model exhibit two equilibra, namely the Rumour Free Equilibrium (RFE) and the Rumour Endemic Equilibrium (REE). Using the method of linearized stability, we establish that the RFE state exist and is locally asymptomatically stable when $R_{0}<1$ and that when $R_{0}>1$ the endemic state exist. The model allows us to discuss the relationship between spreaders and blackmailers, and the effect of blackmailers on the stiflers. Finally, we present numerical simulations that show the impact of political motivated rumours and how its control can be achieved.
\end{abstract}

Keywords: Rumor propagation, Political motivated rumour, Epidemiological models; stability analysis, Transition parameter

\section{INTRODUCTION}

Rumours are an integral part of human life and its spread has significant impact in society. Basically, rumours are unverified stories or report circulating in a community, usually by 
word of mouth or via social network and are accepted as facts, although its original source may be unknown (DiFonzo \& Prashant, 2007). Rumours reflect people desired to find meaning to events around them. Rumours may contain classified information, true or false information about any issue and information that may be detriment to the society at a given time. Various types of rumours exist for various reasons. Some rumours are characterized by the channel of communication, like social network rumours, newspapers rumours, while others are characterized by the classes of people involved and the environment where the thrive, like rumours that spread in an organization (like hospital or schools about management), rumours occurring among specific group of people and political rumours (rumours that spread for political motivated reasons).

The classical model for the spread of rumours was first introduced by Daley and Kandal (1965) and Maki and Thompson (1973). The approach in these models has been used extensively for quantitative studies. In their model, a closed and homogeneous mixed population consisting of three groups; the ignorant, spreaders and the stifler was considered. The ignorant are those individuals who are ignorant of the rumour, the spreader are those who heard the rumour and are actively involved in spreading it and the stifler are those who have heard the rumour but have ceased to spread it. According to them, rumour spread through the law of mass action within the population by a pair-wise contact between spreaders and between spreaders and ignorant. Any spreader involved in a pair-wise contact attempts to "infect" the other individual with the rumour, thereafter individual who was ignorant initially becomes a spreader. In another case, either one or both of those involved in spreading the rumour decide not to tell the rumour to anyone, thereby becoming a stifler (Daley and Gani, 2000). Other non-political rumours models include the works of Oduwole et al.,(2010) who modeled the spread of rumour in an academic institution using markov chain model, Zanette, (2002), Nekovee, et al.,(2007), Chunru and Zujun (2015) and a host of others.

Boris (2002) observed that rumours function as a psychological process that develops around opposing views or mutual misconceptions. One of such psychological function occurs mostly in political circles, which eventually leads to political rumours. According to Weeks and Garrett (2014), political rumours often characterized unsubstantiated claims about candidates and issues that are often false, and are a potentially important source of misperception that may threaten democratic outcomes and objectives. Political rumours arise around topics of national importance and are driven by feeling of uncertainty and anxiety (Rosnow, 1998). This makes it possible for political rumours to flourish during political campaigns by disseminating unverified claims concerning the opposition party or candidates in a bid to mislead the public and affect voting choice (Jamieson, 1992). The spread of political rumour for selfish and negative reasons dates backs to various form of elections in different countries and continents, as seen in the election of Thomas Jefferson as President of the United States (Shibutani, 1996, Weeks \& Garrett, 2014) and has continued in recent election in the United States (Garrett, 2011). This scenario is not different from what is being experienced in recent election in Africa.

According to the theory of partisan motivated reasoning, individuals' prior attitudes will affect how they assess political rumours; hence motivated reasoning suggests that humans evaluate new information in ways that are in line with their prior beliefs (Kunda, 1990; Taber \& Lodge, 2006). This is in consonance with politicians and the political landscape in today modern democracies been practice in different countries and continents. In most political scenarios, individuals are often driven to defend their prior political position 
through biased evaluations of new information. Thus, people are willing to accept attitude consistent to their political position with little evidence while rejecting well supported attitude that are at discrepancy to their political position (Taber \& Lodge, 2006). Recent works on modelling political rumours include those by Sunstein, (2009), Bullock, (2006) and Weeks and Garreth (2014). In this paper, we make a modification to classical model of Huo et. al (2015) by introducing another kind of spreaders called the blackmailers. The new model allows us to discuss the relationship between spreaders and blackmailers (those spreading the rumour for selfish reasons), and the effect of blackmailers on the stiflers. Finally, we present numerical simulations that show the impact of political motivated rumours and how its control can be achieved.

\section{Mathematical Formulation}

The new rumour model is a prototype of the classical susceptible-infected-removed (SIR) model that considers a homogeneous mixing of individuals with state variable as a function of time. The population is sub-divided into five classes; the ignorant class $X(t)$, the latent class, also called the exposed class $W(t)$, true spreader $S(t)$, those spreading the rumour for selfish reasons, also known as the blackmailers $B(t)$ and the stiflers $Z(t)$. The individuals in the ignorant class have not heard the rumour. Individuals in the latent class $W(t)$, have heard the rumour due to contact with the either the true spreader or the blackmailers, but need active effort to discern between true and false rumour and decide to spread the rumour or not; a part of them believe the rumour (either true or false) and decides either to spread it or become stiflers. Those in the spreaders class either continue as true spreaders or as blackmailers (that is, those who spread the rumour for selfish reasons). Both the true spreaders and the blackmailers can become stiflers at a certain time $(t)$ after losing interest in spreading the rumour. The total population at time $t$ is denoted by $N(t)$, where $N(t)=$ $X(t)+W(t)+S(t)+B(t)+Z(t)$. The model diagram is shown in figure 1 below:

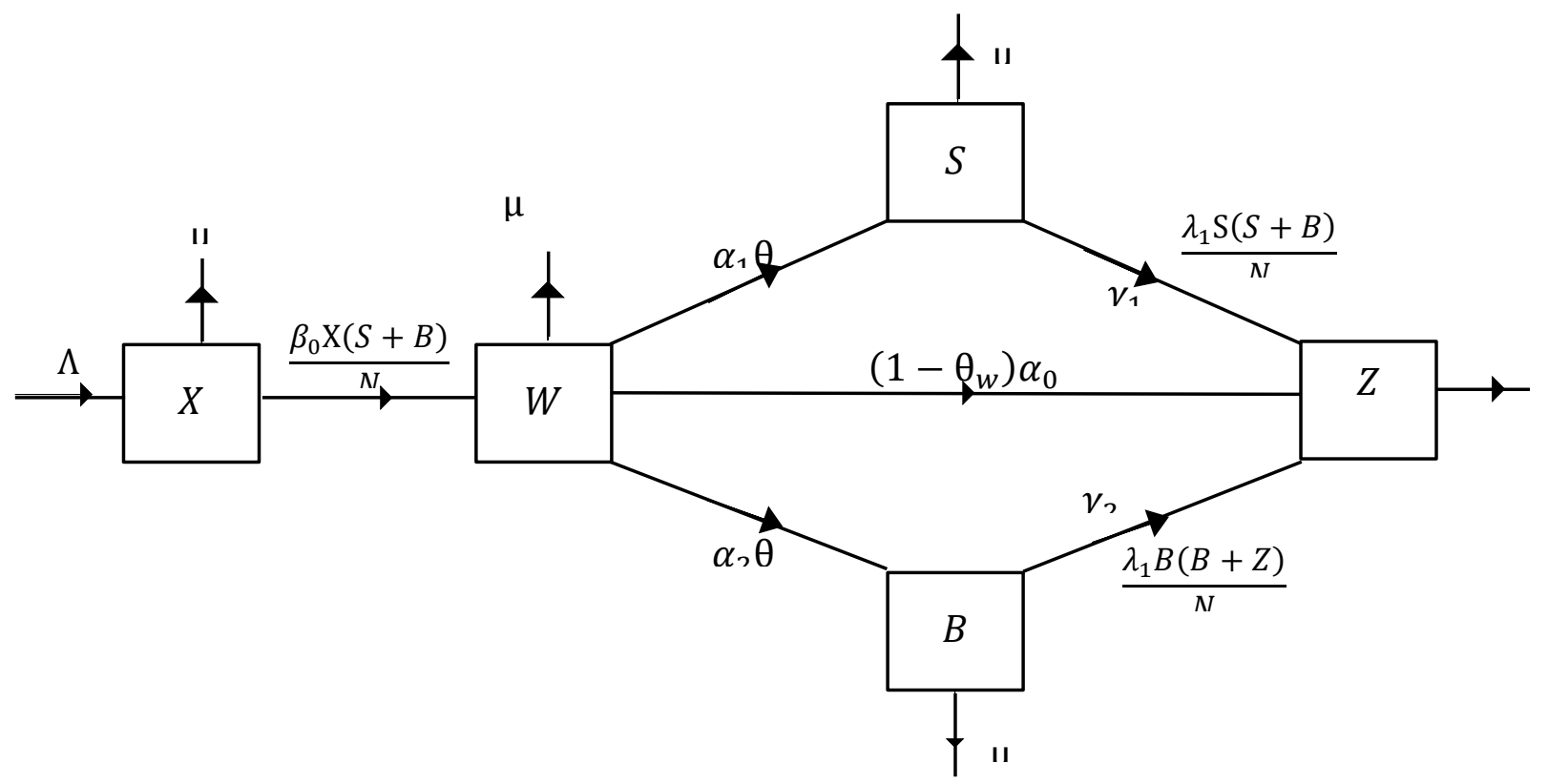

Figure 1. Flow diagram of population dynamics for the spread of rumour

There is no transition of rumour unless a spreader (either in the $S(t)$ or $B(t)$ class) contact an ignorant individual or another spreader. A spreader contacts an ignorant individual and transmits the rumour at a constant frequency and the ignorant individual now move to the latent class or exposed class, where time is require to distinguished between true or false 
rumour content and decides either to be in the $S(t)$ or $B(t)$ class or chose not to spread the rumour and move stifler class $Z(t)$. An ignorant individual become exposed to the rumour at a rate of $\frac{\beta_{0} \mathrm{X}(S+B)}{N}$ within a small interval $(t, t+\Delta t)$, where $\beta_{0}$ is a positive constant number representing the product of the contact frequency and the probability of transmitting the rumour. Those in the exposed class have heard the rumour, but are not yet spreaders. We assume that $\alpha_{0} W$ is the rate at which an individual in the exposed class become spreaders and other become stiflers at the rate of $1-\theta$. Two or more spreaders (true spreader and another true spreader, or true spreader and a blackmailer or a blackmailer and a blackmailer) both transmit the rumour at a constant frequency after which they become bored, losing interest in the rumour and will eventually become stiflers at the rate $\frac{\lambda_{1} \mathrm{~S}(S+B)}{N}$ and $\frac{\lambda_{1} B(B+Z)}{N}$ respectively for true spreaders and blackmailer respectively.

\section{Assumptions:}

a) There is no transition of rumour unless a spreader (either in the $S(t)$ or $B(t)$ class) contact an ignorant individual or another spreader.

b) An Ignorant individual do not always become spreaders (either in the $S(t)$ or $B(t)$ class) initially, but enters into the latent class with conditional transition parameter $\beta_{0}$ and $\theta_{0}=1$

c) A constant immigration rate $(\Lambda)$ and a constant emigration rate in all classes $(\mu)$ are considered.

The table below 1 shows the variables and parameters used in the new model.

Table 1 Model variables and parameters

\begin{tabular}{cl}
\hline Variable & Description \\
\hline$X(t)$ & Number of Ignorant individuals at time $t$ \\
$W(t)$ & Number of Exposed individuals at time $t$ \\
$S(t)$ & Number of true spreaders individuals at time $t$ \\
$B(t)$ & Number of Blackmailers individuals at time $t$ \\
$Z(t)$ & Number of Stiflers individuals at time $t$ \\
\hline Parameter & Description \\
\hline$\beta_{0}$ & Rumour propagation coefficient \\
$\alpha_{0}$ & Rate at which individual at the latent class $(w)$ becomes stiflers \\
$\alpha_{1}$ & Rate at which individual at the latent class $(w)$ becomes true spreaders \\
$\alpha_{2}$ & Rate at which individual at the latent class $(w)$ becomes false spreaders (blackmailers) \\
$\alpha_{s}$ & Change rate for the exposed/latent class $(w)$ who become true spreaders \\
$\alpha_{b}$ & Change rate for the exposed/latent class $(w)$ who becomes false spreaders (blackmailers) \\
$\lambda_{1}$ & True-spreaders stifler coefficient \\
$\lambda_{2}$ & Blackmailers-stifler coefficient \\
$\gamma_{1}$ & Change rate for true-spreaders. \\
$\gamma_{2}$ & Change rate for blackmailers. \\
$\mu$ & Emigration rate \\
$\Lambda$ & Immigration rate \\
$\theta$ & Believe and spread rate, $\theta_{w}=$ Believe and spread rate for the exposed class, $\theta_{w}=1$ \\
& Total population
\end{tabular}

From the assumptions and the flow diagram above, the following model equations are derived.

$\frac{d X}{d t}=\Lambda-\frac{\beta_{0}(S+B) X}{N}-u X$ 


$$
\begin{array}{ll}
\frac{d W}{d t}=\frac{\beta_{0}(S+B) X}{N}-\left(\alpha_{1}+\alpha_{2}\right) \theta W-\mu W-\left(1-\theta_{w}\right) \alpha_{0} W & \ldots \\
\frac{d S}{d t}=\alpha_{1} \theta W-\frac{\lambda_{1}(S+Z) S}{N}-\mu S-\gamma_{1} S & \\
\frac{d B}{d t}=\alpha_{2} \theta W-\frac{\lambda_{2}(B+Z) B}{N}-\mu B-\gamma_{2} B & \ldots \\
\frac{d Z}{d t}=\left(1-\theta_{w}\right) \alpha_{0} W+\frac{\lambda_{1}(S+Z) S}{N}+\frac{\lambda_{2}(B+Z) B}{N}+\gamma_{1} S+\gamma_{2} B-\mu Z & \ldots
\end{array}
$$

We transform the model equations (1) - (5) above to proportion as follows:

Let $x=\frac{X}{N^{\prime}}, w=\frac{W}{N}, \mathrm{~s}=\frac{\mathrm{s}}{N^{\prime}}, b=\frac{B}{N}, z=\frac{Z}{N}$ so that $x+w+s+b+z=1$ and let $\tau=\mu t$, $\lambda_{s}=\frac{\lambda_{1}}{\mu}, \lambda_{b}=\frac{\lambda_{2}}{\mu}, \alpha_{s}=\frac{\alpha_{1}}{\mu}, \alpha_{b}=\frac{\alpha_{2}}{\mu}, \gamma_{a}=\frac{\gamma_{1}}{\mu}, \gamma_{b}=\frac{\gamma_{2}}{\mu}, \beta=\frac{\beta_{0}}{\mu}$

Hence adding equation (1) - (5), we have that

$$
\begin{aligned}
& \frac{d N}{d t}=\frac{d X}{d t}+\frac{d W}{d t}+\frac{d B}{d t}+\frac{d S}{d t}+\frac{d Z}{d t} \\
& \frac{d N}{d t}=\wedge-\frac{\beta_{0}(S+B) X}{N}-u X+\frac{\beta_{0}(S+B) X}{N}-\left(\alpha_{1}+\alpha_{2}\right) \theta W-\mu W-\left(1-\theta_{w}\right) \alpha_{0} W+\alpha_{1} \theta W \\
& -\frac{\lambda_{1}(S+Z) S}{N}-\mu S-\gamma_{1} S+\alpha_{2} \theta W-\frac{\lambda_{2}(B+Z) B}{N}-\mu B-\gamma_{2} B+\left(1-\theta_{w}\right) \alpha_{0} W \\
& +\frac{\lambda_{1}(S+Z) S}{N}+\frac{\lambda_{2}(B+Z) B}{N}+\gamma_{1} S+\gamma_{2} B-\mu Z \\
& \frac{d N}{d t}=\Lambda-\mu(X+W+B+S+Z) \\
& \frac{d N}{d t}=\Lambda-\mu N
\end{aligned}
$$

Therefore,

$\frac{d x}{d t}=\frac{1}{N}\left[\frac{d X}{d t}-x \frac{d N}{d t}\right]$

$\frac{d x}{d t}=\frac{1}{N}\left[\Lambda-\frac{\beta_{0}(S+B) X}{N}-\mu X-\Lambda x+\mu N x\right]$

But $\frac{d x}{d \tau}=\frac{d x}{d t} \cdot \frac{d t}{d \tau}$ and $\frac{d t}{d \tau}=\frac{1}{\mu}$, Then equation (7) becomes

$\frac{d x}{d \tau}=\frac{1}{N}\left[\Lambda-\frac{\beta_{0}(S+B) X}{N}-\mu X-\Lambda x+\mu N x\right] \times \frac{1}{\mu}$

$\frac{d x}{d \tau}=(1-x)-\beta(s+b) x$

Similarly,

$\frac{d w}{d \tau}=\frac{1}{N}\left[\frac{d W}{d t}-w \frac{d N}{d t}\right]$

$\frac{d w}{d t}=\frac{1}{N}\left[\frac{\beta_{0}(S+B) X}{N}-\left(\alpha_{1}+\alpha_{2}\right) \theta W-\mu W-\left(1-\theta_{w}\right) \alpha_{0} W-w(\Lambda-\mu N)\right]$

$\frac{d w}{d \tau}=\frac{d w}{d t} \cdot \frac{d t}{d \tau}$ and $\frac{d t}{d \tau}=\frac{1}{\mu}$, Then equation (9) becomes

$\frac{d w}{d \tau}=\frac{1}{N}\left[\frac{\beta_{0}(S+B) X}{N}-\left(\alpha_{1}+\alpha_{2}\right) \theta W-\mu W-\left(1-\theta_{w}\right) \alpha_{0} W-w(\Lambda-\mu N)\right] \times \frac{1}{\mu}$

$\frac{d w}{d \tau}=\beta(s+b) x-\left(\alpha_{s}+\alpha_{b}\right) \theta w-\left(1-\theta_{w}\right) \alpha_{0} w-w$

But $\theta_{w}=1$, based on the assumption that the latent class will spread the rumour and equation (10) becomes

$\frac{d w}{d \tau}=\beta(s+b) x-\left(\alpha_{s}+\alpha_{b}\right) \theta w-w$

Similarly,

$\frac{d s}{d t}=\frac{1}{N}\left[\frac{d S}{d t}-s \frac{d N}{d t}\right]$ 
$\frac{d s}{d t}=\frac{1}{N}\left[\alpha_{1} \theta W-\frac{\lambda_{1}(S+Z) S}{N}-\mu S-\gamma_{1} S-s(\Lambda-\mu N)\right]$

$\frac{d s}{d \tau}=\frac{d s}{d t} \cdot \frac{d t}{d \tau}$ and $\frac{d t}{d \tau}=\frac{1}{\mu}$, Then equation (12) becomes

$\frac{d s}{d \tau}=\frac{1}{N}\left[\alpha_{1} \theta W-\frac{\lambda_{1}(S+Z) S}{N}-\mu S-\gamma_{1} S-s(\Lambda-\mu N)\right] \times \frac{1}{\mu}$

$\frac{d s}{d \tau}=\theta \alpha_{s} w-\lambda_{s}(s+z) s-\gamma_{s} s-s$

Similarly,

$\frac{d b}{d t}=\frac{1}{N}\left[\frac{d B}{d t}-b \frac{d N}{d t}\right]$

$\frac{d b}{d t}=\frac{1}{N}\left[\alpha_{2} \theta W-\frac{\lambda_{2}(B+Z) B}{N}-\mu B-\gamma_{2} B-b(\Lambda-\mu N)\right] \quad .$.

$\frac{d b}{d \tau}=\frac{d b}{d t} \cdot \frac{d t}{d \tau}$ and $\frac{d t}{d \tau}=\frac{1}{\mu}$, Then equation (14) becomes

$\frac{d b}{d \tau}=\frac{1}{N}\left[\alpha_{2} \theta W-\frac{\lambda_{2}(B+Z) B}{N}-\mu B-\gamma_{2} B-b(\Lambda-\mu N)\right] \times \frac{1}{\mu}$

$\frac{d b}{d \tau}=\theta \alpha_{b} w-\lambda_{b}(b+z) b-\gamma_{b} b-b$

Finally,

$\frac{d z}{d t}=\frac{1}{N}\left[\frac{d Z}{d t}-z \frac{d N}{d t}\right]$

$\frac{d z}{d t}=\frac{1}{N}\left[\left(1-\theta_{w}\right) \alpha_{0} W-\frac{\lambda_{1}(S+Z) S}{N}+\frac{\lambda_{2}(B+Z) B}{N}+\gamma_{1} S+\gamma_{2} B-\mu Z-z(\Lambda-\mu N)\right] .$.

$\frac{d z}{d \tau}=\frac{d z}{d t} \cdot \frac{d t}{d \tau}$ and $\frac{d t}{d \tau}=\frac{1}{\mu}$, Then equation (16) becomes

$\frac{d z}{d \tau}=\frac{1}{N}\left[\left(1-\theta_{w}\right) \alpha_{0} W-\frac{\lambda_{1}(S+Z) S}{N}+\frac{\lambda_{2}(B+Z) B}{N}+\gamma_{1} S+\gamma_{2} B-\mu Z-z(\Lambda-\mu N)\right] \times \frac{1}{\mu}$

$\frac{d z}{d \tau}=\left(1-\theta_{w}\right) \alpha w-\lambda_{s}(s+z) s-\lambda_{b}(b+z) b+\gamma_{s} s+\gamma_{b} b-z \ldots$

Rewriting $\tau$ as $t$ and letting $z=1-x-w-s-b$, equation (8), (11), (13) and (15) becomes

$\frac{d x}{d t}=(1-x)-\beta(s+b) x$

$\frac{d w}{d t}=\beta(s+b) x-\left(\alpha_{s}+\alpha_{b}\right) \theta w-w$

$\frac{d s}{d t}=\theta \alpha_{s} w-\lambda_{s}(1-x-w-b) s-\gamma_{s} s-s$

$\frac{d b}{d \tau}=\theta \alpha_{b} w-\lambda_{b}(1-x-w-s) b-\gamma_{b} b-b$

\section{Mathematical Treatment and Analysis}

\section{Equilibrium States of the Model}

We discuss the Rumour Free Equilibrium (RFE) and the Rumour Endemic Equilibrium (REE) of the model equations (18) - (21). Equating the left hand side of equations (18) - (21) to zero and letting $w=0, s=0, b=0$, we have that

$0=(1-x)$

$x=1$

Hence the Rumour free equilibrium (RFE) is $(1,0,0,0)$

For the Rumour Endemic Equilibrium (REE), we equate equation (18) - (21) to zero, letting $\alpha_{s}+\alpha_{b}=m, s+b=\rho$ and solving simultaneously we have

$\left.\begin{array}{c}x=1-w(m \theta+1) \\ w=\frac{\beta \rho}{\beta \rho(\theta m+1)+m \theta+1} \\ s=\frac{w \theta \alpha_{s}}{\lambda_{s}(1-x-w-s)-\gamma_{s}-1} \\ b=\frac{w \theta \alpha_{b}}{\lambda_{b}(1-x-w-s)-\gamma_{b}-1}\end{array}\right\}$ 


\section{Computation of Basic Reproduction Number $\left(\boldsymbol{R}_{\mathbf{0}}\right)$}

In this section, we compute the Basic Reproduction Number $\left(R_{0}\right)$ using the next generation method as applied by Diekmann et al., and Van den Driessche and Watmough, (2002). From equation (18) - (21) using their approached we have that

$$
\mathcal{F}_{i}=\left(\begin{array}{c}
\beta(s+b) x \\
0 \\
0
\end{array}\right) \text { and } \mathcal{V}_{i}=\left(\begin{array}{c}
m \theta w+w \\
-\theta \alpha_{s} w+\lambda_{s}(1-x-w-b) s+\gamma_{s} s+s \\
-\theta \alpha_{b} w+\lambda_{b}(1-x-w-s) b+\gamma_{b} b+b
\end{array}\right)
$$

where $\mathcal{F}_{i}$ and $\mathcal{V}_{i}$ are the rate of appearances of new infections (rumour) in compartment $i$ and the transfer of individuals into and out of compartment $i$ by all other means respectively. Using the linearization method, the associated matrices at rumour-free equilibrium $(R F E)$ and after taking partial derivatives as defined by

$\mathcal{D F}_{i}\left(E_{0}\right)=\left(\begin{array}{ll}F & 0 \\ 0 & 0\end{array}\right)$ and $\mathcal{D} \mathcal{V}_{i}\left(E_{0}\right)=\left(\begin{array}{ll}V & 0 \\ J_{3} & J_{4}\end{array}\right)$

where $F$ is nonnegative and $V$ is a non-singular matrix, in which both are the $m \times m$ matrices defined by

$F=\left[\frac{\partial \mathcal{F}_{i}}{\partial x_{j}}\left(R F E_{0}\right)\right]$ and $V=\left[\frac{\partial \mathcal{V}_{i}}{\partial x_{j}}\left(R F E_{0}\right)\right]$, with $1 \leq i, j \leq m$ and $m$ is the number of infected classes.

In particular $m=3$, we have

$F=\left(\begin{array}{lll}0 & 0 & 0 \\ \beta & 0 & 0 \\ \beta & 0 & 0\end{array}\right)$ and $V=\left(\begin{array}{ccc}m \theta+1 & -\theta \alpha_{s} & -\theta \alpha_{b} \\ 0 & \gamma_{s}+1 & 0 \\ 0 & 0 & \gamma_{b}+1\end{array}\right)$

If the inverse of $V$ is given as

$$
V^{-1}=\left(\begin{array}{ccc}
\frac{1}{m \theta+1} & \frac{\theta \alpha_{s}}{\gamma_{s}+1} & \frac{\theta \alpha_{b}}{\gamma_{b}+1} \\
0 & \frac{1}{\gamma_{s}+1} & 0 \\
0 & 0 & \frac{1}{\gamma_{b}+1}
\end{array}\right)
$$

Then the next generation matrix denoted by $F V^{-1}$ is given as

$$
F V^{-1}=\left(\begin{array}{ccc}
0 & 0 & 0 \\
\frac{\beta}{m \theta+1} & \frac{\beta \theta \alpha_{s}}{\gamma_{s}+1} & \frac{\beta \theta \alpha_{b}}{\gamma_{b}+1} \\
\frac{\beta}{m \theta+1} & \frac{\beta \theta \alpha_{s}}{\gamma_{s}+1} & \frac{\beta \theta \alpha_{b}}{\gamma_{b}+1}
\end{array}\right)
$$

We find the eigenvalues of $F V^{-1}$ by setting the determinant $\left|F V^{-1}-\eta I\right|=0$

$$
\left|F V^{-1}-\eta I\right|=\left|\begin{array}{ccc}
-\eta & 0 & 0 \\
\frac{\beta}{m \theta+1} & \frac{\beta \theta \alpha_{s}}{\gamma_{s}+1}-\eta & \frac{\beta \theta \alpha_{b}}{\gamma_{b}+1} \\
\frac{\beta}{m \theta+1} & \frac{\beta \theta \alpha_{s}}{\gamma_{s}+1} & \frac{\beta \theta \alpha_{b}}{\gamma_{b}+1}-\eta
\end{array}\right|=0
$$

with characteristics polynomial

$$
\rho(\eta)=-\eta^{3}+\frac{\beta \theta \alpha_{s}\left[\left(\gamma_{b}+1\right) \alpha_{s}+\left(\gamma_{s}+1\right) \alpha_{b}\right]}{\left(\gamma_{s}+1\right)\left(\gamma_{b}+1\right)} \eta^{2}-\frac{2 \beta^{2} \theta^{2} \alpha_{s} \alpha_{b}}{\left(\gamma_{s}+1\right)\left(\gamma_{b}+1\right)} \eta
$$

and characteristics equation given as

$-\eta^{3}+\frac{\beta \theta \alpha_{s}\left[\left(\gamma_{b}+1\right) \alpha_{s}+\left(\gamma_{s}+1\right) \alpha_{b}\right]}{\left(\gamma_{s}+1\right)\left(\gamma_{b}+1\right)} \eta^{2}-\frac{2 \beta^{2} \theta^{2} \alpha_{s} \alpha_{b}}{\left(\gamma_{s}+1\right)\left(\gamma_{b}+1\right)} \eta=0$ 
Solving the characteristic equation (26) for the eigenvalues $\eta_{1}, \eta_{2}$, and $\eta_{3}$, we obtained $\eta_{1}=\eta_{2}=0$, and $\eta_{3}=\frac{\beta \theta\left(\gamma_{s} \alpha_{b}+\alpha_{b}+\alpha_{s}+\alpha_{s} \gamma_{b}\right)}{\left(\gamma_{s}+1\right)\left(\gamma_{b}+1\right)}$

$R_{0}$ is the maximum of the three eigenvalues $\eta_{1}, \eta_{2}$ and $\eta_{3}$. Hence the Basic Reproductive number is the dominant eigenvalues of $F V^{-1}$. Thus we have that

$R_{0}=\frac{\beta \theta\left(\gamma_{s} \alpha_{b}+\alpha_{b}+\alpha_{s}+\alpha_{s} \gamma_{b}\right)}{\left(\gamma_{s}+1\right)\left(\gamma_{b}+1\right)}$

\section{Stability Analysis of the Rumour Free Equilibrium of the Model}

To study the behaviour of the system (18) - (21) around the Rumour-free equilibrium state matrix at $R F E=[1,0,0,0]$, we resort to the linearized stability approach.

Let

$f_{1}=(1-x)-\beta(s+b) x$

$f_{2}=\beta(s+b) x-\left(\alpha_{s}+\alpha_{b}\right) \theta w-w$

$f_{3}=\theta \alpha_{s} w-\lambda_{s}(1-x-w-b) s-\gamma_{s} s-s$

$f_{4}=\theta \alpha_{b} w-\lambda_{b}(1-x-w-s) b-\gamma_{b} b-b$

$$
J_{R F E}=\left[\begin{array}{cccc}
-1 & 0 & -\beta & -\beta \\
0 & -\left[\left(\alpha_{s}+\alpha_{b}\right) \theta+1\right] & \beta & \beta \\
0 & \theta \alpha_{s} & -\left(\gamma_{s}+1\right) & 0 \\
0 & \theta \alpha_{s} & 0 & -\left(\gamma_{b}+1\right)
\end{array}\right]
$$

The Determinant and the Trace of the Jacobian matrix of equation (32) is given as

$\operatorname{Det}\left(J_{R F E}\right)=\beta \theta\left(\alpha_{b}+\alpha_{b} \gamma_{b}+\alpha_{s}+\alpha_{s} \gamma_{b}\right)+m \theta+\left(1+\gamma_{s}\right)\left(1+\gamma_{b}\right) \ldots$ (33)

$\operatorname{Trace}\left(J_{R F E}\right)=-\left(4+\alpha_{s} \theta+\alpha_{b} \theta+\gamma_{s}+\gamma_{b}\right)$

\section{Theorem 1}

The Rumour free equilibrium state $R F E=[1,0,0,0]$ of the model (18) - (21) is locally asymptotically stable if $R_{0}<1$, otherwise $R F E$ is unstable.

\section{Proof}

Then Jacobian matrix $\left(U_{R F E}\right)$ of the model equations (18) - (21)at the RFE is given by

$$
J_{R F E}=\left[\begin{array}{cccc}
-1 & 0 & -\beta & -\beta \\
0 & -\left[\left(\alpha_{s}+\alpha_{b}\right) \theta+1\right] & \beta & \beta \\
0 & \theta \alpha_{s} & -\left(\gamma_{s}+1\right) & 0 \\
0 & \theta \alpha_{s} & 0 & -\left(\gamma_{b}+1\right)
\end{array}\right]
$$

If the Jacobian matrix is evaluated at the Rumour-Free equilibrium state (RFE), then the required criteria for stable equilibrium are that the Determinant of the Jacobian is positive and the Trace of the Jacobian is negative.

From the Determinant and the Trace of the Jacobian matrix of equation (32), we have that $\operatorname{Det}\left(J_{R F E}\right)=-\beta \theta \alpha_{s}\left(\gamma_{b}+\gamma_{s}+2\right)+m_{1} \theta+\left(1+\gamma_{s}\right)\left(1+\gamma_{b}\right)$

where $m_{1}=\gamma_{b}\left(\alpha_{b} \gamma_{s}+\alpha_{s} \gamma_{s}+\alpha_{b}+\alpha_{s}\right)+\left(\alpha_{b}+\alpha_{s}\right)\left(\gamma_{s}+1\right)$

$\operatorname{Det}\left(J_{R F E}\right)=\left(1+\gamma_{s}\right)\left(1+\gamma_{b}\right)+m_{1} \theta-R_{0} \alpha_{s}$

$\operatorname{Det}\left(J_{R F E}\right)=\left(1+\gamma_{s}\right)\left(1+\gamma_{b}\right)+m_{1} \theta-R_{0} \alpha_{s}>0$ provided that $R_{0}<1$

Also

$\operatorname{Trace}\left(J_{R F E)}=-\left(4+\alpha_{s} \theta+\alpha_{b} \theta+\gamma_{s}+\gamma_{b}\right)<0\right.$

$\operatorname{Trace}\left(J_{R F E)}=-\left(\frac{4 \beta+R_{0}\left(1+\gamma_{s}\right)\left(1+\gamma_{b}\right)+\beta \gamma_{s}\left(1+\theta \alpha_{b}\right)+\beta \gamma_{b}\left(1+\theta \alpha_{s}\right)}{\beta}\right)<0\right.$

$\operatorname{Trace}\left(J_{R F E)}=-\left(4+\frac{R_{0}}{\beta}\left(1+\gamma_{s}\right)\left(1+\gamma_{b}\right)+\gamma_{s}\left(1+\theta \alpha_{b}\right)+\gamma_{b}\left(1+\theta \alpha_{s}\right)\right)<0\right.$

$\operatorname{Trace}\left(J_{R F E)}=-\left(4+\frac{R_{0}}{\beta}\left(1+\gamma_{s}\right)\left(1+\gamma_{b}\right)+m_{2}\right)<0\right.$ 
where $m_{2}=\gamma_{s}\left(1+\theta \alpha_{b}\right)+\gamma_{b}\left(1+\theta \alpha_{s}\right)$

$\operatorname{Trace}\left(J_{R F E}\right)=-\left(4+\frac{R_{0}}{\beta}\left(1+\gamma_{s}\right)\left(1+\gamma_{b}\right)+m_{2}\right)<0$, provided that $R_{0}<1$

\section{RESULTS}

\section{Numerical Experiments of the Model}

The Rumour propagation model (18) - (21) was solved numerically using Runge-KuttaFehllberg4th order method and implemented using Maple 17 (Maplesoft, Waterloo)

The following experiments were carried out

Experiment 1: Effect of prevalence of rumour spread by $S(t)$ and $B(t)$ with constant transition rate $\alpha_{1}=\alpha_{2}$ when $\gamma_{1}>\gamma_{2}$ (Case 1$)$ and $\gamma_{1}<\gamma_{2}$ (Case 2$)$

Experiment 2: Effect of prevalence of rumour spread by $S(t)$ and $B(t)$ with variable transition rate $\alpha_{1}<\alpha_{2}$ when $\gamma_{1}>\gamma_{2}$ (Case 1) and $\gamma_{1}<\gamma_{2}$ (Case 2)

Experiment 3: Effect of prevalence of rumour spread by $S(t)$ and $B(t)$ with variable transition rate $\alpha_{1}>\alpha_{2}$ when $\gamma_{1}<\gamma_{2}$ (Case 1) and $\gamma_{1}>\gamma_{2}$ (Case 2)

Table 2 Estimated values of the parameters used for simulation of the rumour model

\begin{tabular}{|c|c|c|c|c|c|c|c|}
\hline Parameter & $x(0)$ & $w(\mathbf{0})$ & $s(\mathbf{0})$ & $b(\mathbf{0})$ & $\beta_{0}$ & $\mu$ & $\Lambda$ \\
\hline Values & 1.0 & 0.5 & 0.2 & 0.2 & 0.08 & 0.1 & 0.1 \\
\hline Sources & Assumed & Assumed & Assumed & Assumed & Assumed & $\begin{array}{l}\text { Huo et al } \\
\text { (2015) }\end{array}$ & $\begin{array}{l}\text { Huo et al } \\
\text { (2015) }\end{array}$ \\
\hline Parameter & $\theta$ & $\alpha_{1}$ & $\alpha_{2}$ & $\lambda_{1}$ & $\lambda_{2}$ & $\gamma_{1}$ & $\gamma_{1}$ \\
\hline Values & 0.7 & $0.11^{\text {** }}$ & $0.11^{* *}$ & $0.2^{* *}$ & 0.2 & 0.08 & 0.05 \\
\hline Sources & $\begin{array}{l}\text { Huo et al } \\
\text { (2015) }\end{array}$ & Assumed & Assumed & $\begin{array}{l}\text { Huo et al } \\
\text { (2015) }\end{array}$ & Assumed & Assumed & $\begin{array}{l}\text { Huo et al } \\
\text { (2015) }\end{array}$ \\
\hline
\end{tabular}

Assumed: Hypothetical data use for research purpose. ${ }^{* *}$ Based on parameter value 
Experiment 1 Case 1, $\alpha_{1}=\alpha_{2}, \gamma_{1}>\gamma_{2}$

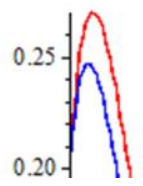

Experiment 2 Case 1, $\alpha_{1}<\alpha_{2}, \gamma_{1}>\gamma_{2}$

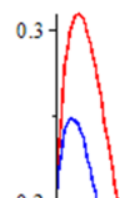

Experiment 3 Case 1, $\alpha_{1}>\alpha_{2}, \gamma_{1}<\gamma_{2}$

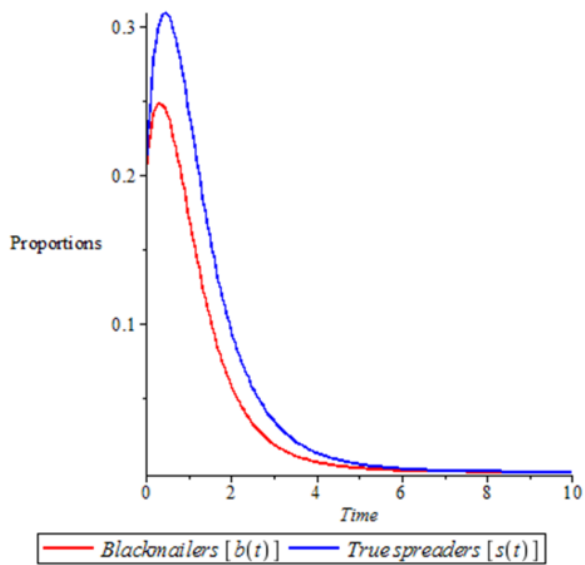

Experiment 1 Case 2, $\alpha_{1}=\alpha_{2}, \gamma_{1}<\gamma_{2}$

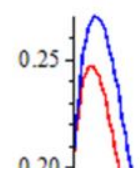

Experiment 2 Case 2, $\alpha_{1}<\alpha_{2}, \gamma_{1}<\gamma_{2}$

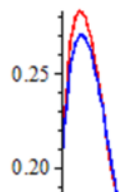

Experiment 3 Case 2, $\alpha_{1}>\alpha_{2}, \gamma_{1}>\gamma_{2}$

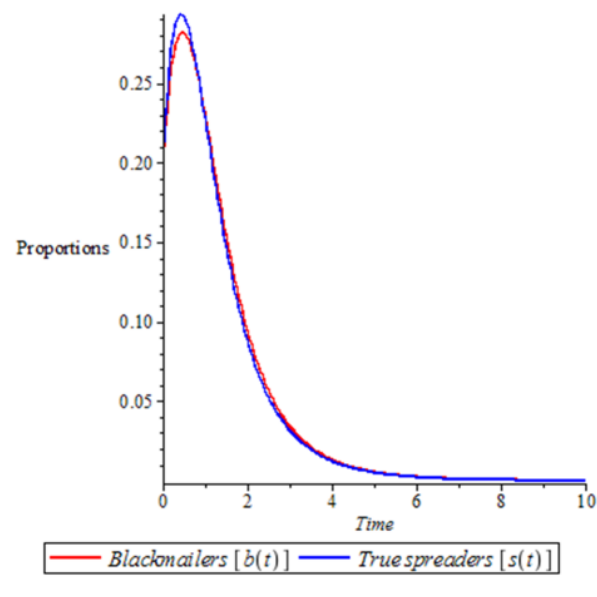

Figure 4 Effect of prevalence of rumour spread by $S(t)$ and $B(t)$ with variable transition rate $\alpha_{1}>\alpha_{2}$ when $\gamma_{1}<\gamma_{2}$ (Case 1) and $\gamma_{1}>\gamma_{2}$ (Case 2)

spreaders, and the contents in the rumour spread by biackmailers will stay on tor some time before diminishing. The change rate for true-spreaders is the rate at which the a member in the true-spreaders class $S(t)$ becomes a blackmailers $B(t)$ or a stifler $Z(t)$. This is similar to the second observation in case 2 , when the change rate of true-spreaders is less than the change rate of blackmailers leading to an increase in the spread of rumour content of the true-spreaders $S(t)$ class. Generally, both the members in the exposed class $W(t)$ and the stifler $Z(t)$ becomes either a true-spreader when being convinced of the truth of the rumor and then decides to inform others or a blackmailer by twisting the contents of the unverified information for selfish reason and then decides to spread the rumour. It should be noted that a member of the exposed class after coming in contact with the rumour from either a true spreader or a blackmailer can possibly refuse to spread the rumor, or alternately a spreader (true-spreaders or blackmailers) can lose interest in the rumor and then decide not to spread the rumor any further. Hence the controlling parameter must be able to change the believe concept of the person carrying the rumour. 
In figure 3, we examined the effect of prevalence of rumour spread by true-spreaders $S(t)$ and blackmailers $B(t)$ when there is a varying transition rate $\left(\alpha_{1}<\alpha_{2}\right)$. We observed that when the change rate of true-spreaders $\left(\gamma_{1}\right)$ is greater than the change rate of blackmailers $\left(\gamma_{2}\right)$, the frequency of the rumour content spread by blackmailers is high, but when the change rate of true-spreaders $\left(\gamma_{1}\right)$ is less than the change rate of blackmailers $\left(\gamma_{2}\right)$, then the rumour frequency between both populations is reasonably control. This infers to the allusion that political opponents must learn to respond or counteract their opponent view before the public.

According to Huo, et. al (2015), individuals in the exposed/latent and stiflers class must be provided with clear background knowledge that will assist them raise some reasonable questions in order to present logical arguments in order to assess the credibility or the validity of the rumour as they come in contact with either the true-spreaders or with the blackmailers. This form of knowledge is a determining factor that contributes to the termination of the spread of rumour in political environment.

In figure 4, we examined the effect of prevalence of rumour spread by true-spreaders $S(t)$ and blackmailers $B(t)$ when there is a varying transition rate $\left(\alpha_{1}>\alpha_{2}\right)$. We observed that when the change rate of true-spreaders $\left(\gamma_{1}\right)$ is less than the change rate of blackmailers $\left(\gamma_{2}\right)$, the frequency of the rumour content spread by blackmailers is low, but when the change rate of true-spreaders $\left(\gamma_{1}\right)$ is greater than the change rate of blackmailers $\left(\gamma_{2}\right)$, then the rumour frequency between both populations is reasonably control.

According to Kostka et al. (2008), the starting point for rumor dissemination in the society must be identified. According Kostka et al. (2008)), different starting point in the dynamic of rumour network leads to different spreading behavior for a rumor. For our discussion on political rumour have interesting starting point during election seasons and to score political antagonism among the political class. Also it should be noted that the structure of society and its homogeneity are two important factors on how rumor statements mutate and how many people can hear and change the propositions of the rumor, and this might lead to an equilibrium point between the true-spreaders and the blackmailers. Therefore, rumor of spreading in a society can reveal belief indicators of the society, especially in the political arena.

From the foregoing we have clearly seen that the controlling parameter in political rumours must go around factors that affect the rate at which individual in the latent class become either a true spreader or a blackmailer that distort information for selfish reasons. These parameters $\alpha_{1}$ and $\alpha_{2}$, together with $\gamma_{1}$ and $\gamma_{2}$ are of great importance in controlling political rumours. The rate of all these parameter are govern by the believe rate $\theta$. There are certain factors that determine the believe rate of an individual in the population. These include the psychology of the hearers in line with his/her political leaning, educational background, benefits of accepting the rumour as true (and for blackmailer) and ability to cipher the intentions of the source of the unverified information. Naturally, people are willing to accept attitude consistent to their political position with little evidence while rejecting well supported attitude that are at discrepancy to their political position, nevertheless logical reasons with verifiable fact and evidence must substantiate and rebuff all false rumour.

Politicians must learn to use all available means including democratic institutions to set all record right when unverified information about the actions of the government of the day or 
its actors in terms of their personal involvements or activities in government to neutralize the negative impact of political motivated rumours or fake news.

While we do not obtain the optimal control parameter for this model in terms of equations or formula, we have nevertheless shows the impact of political motivated rumours and how it control can be achieve.

\section{CONCLUSION}

Rumours are an integral part of human life and its spread has significant impact in society. Political rumours in recent times are negatively affecting the society and if not check can lead to greater problems in the future. We have consider a rumour propagation model with conditional latent period and varying population is considered. In our model we introduce a new compartment called the blackmailers, another type of spreaders who spread the rumour for selfish reason. There is a direct relationship between true-spreaders and blackmailers. The model exhibit two equilibra, namely the rumour free equilibrium (RFE) and the rumour endemic equilibrium (REE). Using the method of linearized stability, we establish that the RFE state exist and is locally asymptomatically stable when $R_{0}<1$, thus helping us control the spread of the rumour and that when $R_{0}>1$ the endemic state exist leading to the rumour persisting indefinitely through-out the political space.

Political rumours must not be left without providing measures to control it in the political space. Political opponents must learn to use democratic institution to control the negative impact or political motivated rumour. Though acceptance of rumour in the political arena is largely a function of political attachment, there is still the need to use available means to verify the unverified information. Every member of the population including the agencies that spread information must do so with high level of professionalism and ethic.

\section{REFERENCES}

Boris, K. (2002). Simple gossiping with balls and bills> Technical Report for Department of Computing Science, Chalmers University of Technology and Goteberg University, Swedem 212pp

Bullock, J. (2006): "The Enduring Importance of False Political Beliefs" Paper presented at the annual meeting of the Western Political Science Association, Hyatt Regency Albuquerque, Albuquerque, New Mexico Communication, 38, 12-28.

Chunru, Li and Zujun, Ma (2015); Dynamic analysis of a spatial diffusion rumor propagation model with delay. Advances in Difference Equations 2015:364 DOI 10.1186/s13662-015-0655-8

Daley, D. J. and Gani, J. (2000): Epidemic Modelling. Cambridge University Press, Cambridge

Daley, D. J. and Kendall, D. G. (1965): Stochastic rumours. J. Inst. Math. Appl. 1, 42-55

DiFonzo, N. and Prashant B. (2007). Rumor Psychology: Social and Organizational Approaches. Washington, D.C.: American Psychological Association

Garrett, R. K. (2011). Troubling consequences of online political rumoring. Human Communication Research, 37, 255-274

Huo, L., Lin, T., Fan, C., Liu, C. amd Zhao, J (2015): Optimal control of a rumor propagation model with latent period in emergency event. Advances in Difference Equations, 54 DOI 10.1186/s13662-015-0394-x

Jamieson, K. H. (1992). Dirty politics: Deception, distraction, and democracy. New York: Oxford University Press. 
Kunda, Ziva. 1990. “The Case for Motivated Reasoning." Psychological Bulletin 8(3) 480-498

Maki, D. P. and Thompson, M. (1973): Mathematical Models and Applications, with Emphasis on Social, Life, and Management Sciences. Prentice-Hall, Englewood Cliffs

Nekovee, M., Moreno, Y., Bianconi, G., Marsili, M (2007): Theory of rumour spreading in complex social networks. Physica A 374(1), 457-470,. DOI 10.1016/ j.physa.2006.07.017

Oduwole, H. K., Ndakwo, H. S and Okoro, S. I. (2010) Modeling the Spread of Rumour in an Academic Institution via Absorbed Markov chain. Nigerian Journal of Pure and Applied Sciences, Vol. 4, 107-117

Rosnow, R. L. (1988). Rumor as communication: A contextualist approach. Journal of Communication, 38, 12-28.

Shibutani, T. (1966): Improvised News: A Sociological Study of Rumor. Bobbs-Merrill, Indianapolis, America

Sunstein, Cass R. and Adrian Vermeule. 2009. "Conspiracy Theories: Causes and Cures." Journal of Political Philosophy. 17(2): 202-227

Taber, Charles S., and Milton Lodge. 2006. "Motivated Skepticism in the Evaluation of Political Beliefs." American Journal of Political Science 50(3): 755-769.

Weeks, B, E and Garrett R. K. (2014) Electoral Consequences of Political Rumors: Motivated Reasoning, Candidate Rumors, and Vote Choice during the 2008 U.S. Presidential Election. International Journal of Public Opinion Research. doi:10.1093/ijpor/edu005

Zanette, D. H. (2002); Dynamics of rumor propagation on small-world networks. Phys. Rev.

E 65, 4041908. 